\title{
Er:YGG Planar Waveguide Amplifiers for LIDAR Applications
}

\author{
Stephen J. Beecher, James A. Grant-Jacob, Ping Hua, David P. Shepherd, Robert W. Eason and Jacob I. \\ Mackenzie \\ Optoelectronics Research Centre, University of Southampton, SO17 1BJ, UK \\ S.J.Beecher@soton.ac.uk
}

\begin{abstract}
Er:YGG is a promising candidate for greenhouse-gas LIDAR due to fortuitous transitions around key absorption lines of carbon-dioxide and methane. We present growth of crystal films by pulsed-laser-deposition, their spectroscopy and initial characterization as amplifiers.

OCIS codes: (310.0310) Thin films; (220.4610) Optical fabrication; (230.7390) Waveguides, planar; (130.0130) Laser materials; (140.5680) Rare earth and transition metal solid-state lasers.
\end{abstract}

\section{Introduction}

LIDAR (LIght Detection And Ranging) from earth orbiting satellites provides a route to mapping, with unprecedented detail, the creation, movement and storage of various gas species in our atmosphere. For two key green-house gases, $\mathrm{CO}_{2}$ and methane, it is possible to exploit LIDAR in the 1.6-micron regime where there are suitable absorption features and good photodetectors. Nonetheless, the spectral requirements on the laser source are very demanding, including tunable narrow linewidths and high peak powers to obtain good signal fidelity over the relatively long distances from the satellites to ground. Furthermore, there are limited direct emission techniques that enable the coverage of the appropriate wavelengths for the respective gas species $\left(\mathrm{CO}_{2}-1572 \mathrm{~nm}\right.$ and Methane $1651 \mathrm{~nm}$ ). Erbium doped materials, particularly glass, have been used for eye-safe LIDAR systems in the past, although these are typically limited to wavelengths $<1560 \mathrm{~nm}$ [1]. Crystalline hosts offer the potential for higher gains at longer wavelengths but only for discrete lines. The crystal Er:YGG, an isomorph of YAG, possesses spectroscopic properties suited for both $\mathrm{CO}_{2}$ and methane, as shown in Fig. 1. Planar waveguide amplifiers (PWAs) provide a route for achieving high gains in compact devices, through their ability to maintain strong population inversions over longer distances than possible in bulk systems, and in a MOPA configuration could enable direct amplification of low-powered single-frequency diode lasers to the required power levels. We have grown, for what we believe is for the first time, an erbium-doped YGG crystal planar waveguide. Preliminary analysis of the active core, demonstrates we have a high-quality Er-doped YGG crystal, with single-pass internal gain measurements realized at $1533 \mathrm{~nm}$ and $1572 \mathrm{~nm}$, which with improved waveguide quality and increased pump power will enable these devices for LIDAR applications.

\section{Fabrication}

Crystal growth was conducted using the same pulsed laser deposition apparatus discussed in detail in [2]. A $248 \mathrm{~nm}$ $\mathrm{KrF}$ laser was focused onto a pressed ceramic target with a fluence of $1.1 \mathrm{~J} / \mathrm{cm}^{2}$. The target contained a mixture of the oxides of yttrium, gallium and erbium in an atomic ratio 2.973:5:0.027, allowing for the growth of 0.9-at.\% Er:YGG if perfect stoichiometric transfer of material from the target to the substrate occurs. The substrate was a $10 \mathrm{~mm} \times 10 \mathrm{~mm} \times 1 \mathrm{~mm}$ piece of $\langle 100\rangle$ cut YAG and was heated from the back side by a $\mathrm{CO}_{2}$ laser, leading to an estimated substrate temperature of $800{ }^{\circ} \mathrm{C}$. Two hours of deposition led to a film thickness of $9.0 \mu \mathrm{m}$, which was suitably thick for low-loss coupling to conventional single-mode telecoms fiber. After growth, two opposing $10 \mathrm{~mm}$ $\mathrm{x} 1.01 \mathrm{~mm}$ sample facets were lapped and polished plane-parallel, resulting in a waveguide length of $9.0 \mathrm{~mm}$.

\section{Spectroscopy}

To assess the spectroscopy of this relatively uncommon crystal, we conducted fluorescence measurements for emission from the ${ }^{4} \mathrm{I}_{13 / 2}$ level using both excitation to the ${ }^{4} \mathrm{I}_{11 / 2}$ level and in-band pumping. For fluorescence lifetime measurements, a fiber-coupled $969 \mathrm{~nm}$ laser diode, operated in pulsed mode, was focused to a low irradiance $\left(\sim 100 \mathrm{~W} / \mathrm{cm}^{2}\right)$ onto the large-area face of the film. The emission from the film was passed through a silicon filter to remove contribution from the pump light and focused onto an InGaAs photodiode connected to a low noise current amplifier with the waveform was captured using an oscilloscope. The fluorescence decay was well fitted by a single exponential with a decay time of $4.8 \mathrm{~ms}$ (see figure 1). This implies that even at this dopant concentration some quenching of the upper-state lifetime is occurring in comparison to the results of Stange et al. who report a lifetime 
of $5.7 \mathrm{~ms}$ for 0.5 -at.\% samples [3]. Using this setup, but under continuous-wave pumping, the florescence spectra over the 1400-1700 nm range $\left({ }^{4} \mathrm{I}_{13 / 2} \rightarrow{ }^{4} \mathrm{I}_{15 / 2}\right)$ was captured using an optical spectrum analyzer (OSA). Further measurements of the fluorescence spectrum were taken with in-band pumping using a single-mode pump diode producing up to $400 \mathrm{~mW}$ at $1460 \mathrm{~nm}$. This diode was launched via proximity coupling of the single-mode fiber to the polished waveguide facet, with a multimode $(\varnothing=62.5 \mu \mathrm{m}, 0.22 \mathrm{NA})$ fiber placed orthogonal to the plane of the waveguide used to collect fluorescence and deliver it to the OSA. As previously discussed [2], this measurement geometry allows for collection of fluorescence from a small area of the waveguide and minimizes the effect of reabsorption on the fluorescence spectrum. A calculation of the emission cross section for Er:YGG was made using the Füchtbauer-Ladenburg equation. We approximate the radiative quantum efficiency of the upperstate to be 0.69 based on the ratio of our measured fluorescence lifetime and the calculated intrinsic lifetime of $7 \mathrm{~ms}$ from Ashurov et al. [4]. For wavelengths below $1465 \mathrm{~nm}$ the fluorescence from the sample pumped at $970 \mathrm{~nm}$ was used and this was merged with the fluorescence captured using in-band pumping for the longer wavelength region. A resolution of $2 \mathrm{~nm}$ for the measurements below $1465 \mathrm{~nm}$ and $1 \mathrm{~nm}$ for the longer wavelengths is used, but higher resolution $(0.2 \mathrm{~nm})$ measurements of the $1533 \mathrm{~nm}$ fluorescence peak showed no further narrowing of this feature. Of note is the visibility of weak emission in the 1543-1558 nm spectral region. From the reports of Ashurov et al. [4], these features do not belong to $\mathrm{Er}^{3+}$ ions located on the N-centre of the d-site of the lattice (Y substitution) but rather to $\mathrm{Er}^{3+}$ occupying A-centres, corresponding to the $\mathrm{Er}^{3+}$ ion substituting onto one of the $\mathrm{Ga}^{3+}$ sites. This effect is likely made more pronounced for these crystals grown by PLD from a stoichiometric target composition, indeed the film composition was determined by Energy-Dispersive X-ray (EDX) analysis to be slightly deficient in Ga, with potential for these vacancies to be filled by $\mathrm{Er}^{3+}$. The difference in atomic mass between $\mathrm{Y}$ and $\mathrm{Ga}$, results in increased Ga scattering as the plume propagates towards the substrate, leading to an excess of Y. This effect may be further compounded by the higher volatility of Ga in comparison to Y leading to Ga 'boil-off' from the substrate. We have observed similar behaviour in YAG growth, which have been solved by pre-compensation of the target with additional $\mathrm{Al}_{2} \mathrm{O}_{3}$. To calculate absorption cross sections, we use the reciprocity technique based on the energy levels of the $\mathrm{N}$-center reported by Ashurov et al. [4]. These absorption and emission cross sections for the ${ }^{4} \mathbf{I}_{13 / 2}{ }^{->}$ ${ }^{4} \mathbf{I}_{15 / 2}$ transition are shown in Fig. 1.
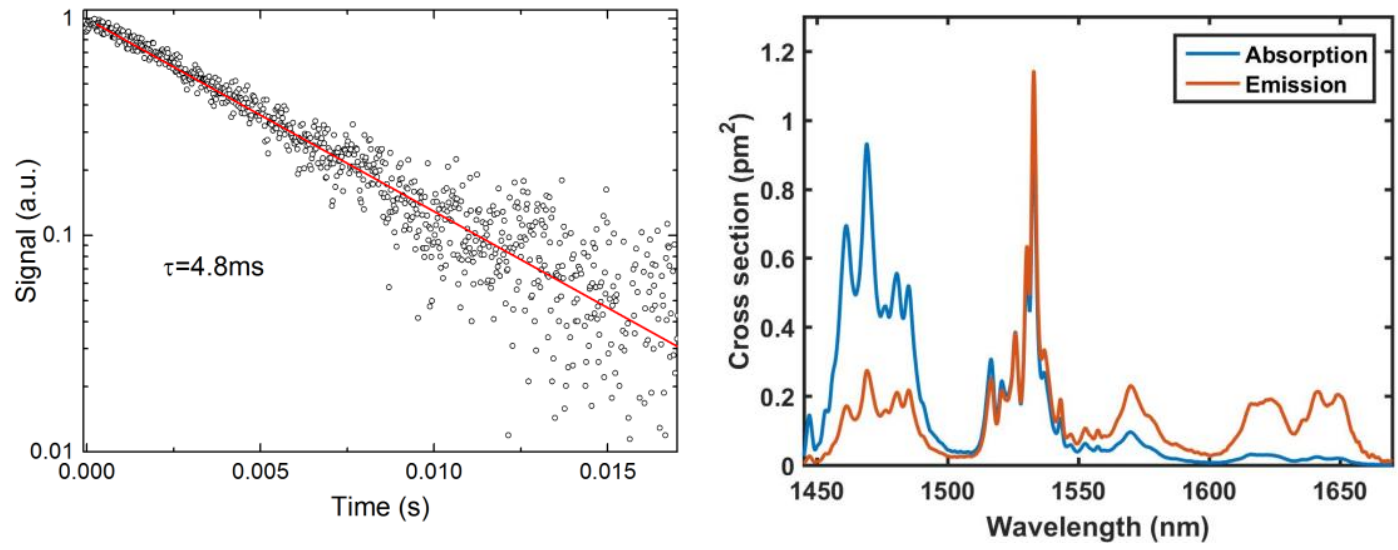

Fig. 1. (Left) Fluorescence lifetime of the Er:YGG. (Right) Calculated absorption and emission cross sections.

\section{Insertion loss and gain measurements}

For insertion loss and gain measurements light from a tunable diode laser (Agilent 81689A) and the 400 mW, $1460 \mathrm{~nm}$ pump diode were brought into the same single-mode fiber using a wavelength division multiplexer (WDM). The output of the WDM was proximity coupled into Corning HI1060 fiber having a mode field diameter of $9.9 \mu \mathrm{m}$ at $1550 \mathrm{~nm}$. The light exiting this fiber was then proximity coupled into the planar waveguide. Light from the unguided waveguide axis was collected and collimated using an $\mathrm{f}=10 \mathrm{~mm}$ cylindrical lens and light from the guided axis was collected and collimated using an $\mathrm{f}=12 \mathrm{~mm}$ lens, resulting in a near symmetric and well-collimated output. Insertion loss measurements, a comparison between the signal power measured after the collimating optics when the waveguide was in place and when the waveguide was removed (with the two cylindrical lenses being repositioned to directly recollimate the fiber output), were taken at seven different wavelengths across the C- and Lbands. These measurements were used along with the calculated spectroscopy from Fig. 1, to calculate the background insertion loss (the insertion loss of the guide in the absence of absorption) and the dopant concentration in a manner similar to that reported by Parsonage et al. [5]. The results from these measurements and the fit to this 
data are displayed in Fig. 2, yielding a background insertion loss of $1.95 \mathrm{~dB}$ and an Er ion concentration of $0.9 \times 10^{20}$ ions per $\mathrm{cm}^{3}$ corresponding to a 0.7 at.\% doping level. It should be noted that this doping level value only includes the $\mathrm{N}$-centre ions, which dominate the absorption and emission characteristics. For the background insertion-loss value we attribute $0.90 \mathrm{~dB}$ to Fresnel reflections from the two air-waveguide interfaces; this could be significantly decreased to $<0.01 \mathrm{~dB}$ by suitable anti-reflection coatings. We further attribute $0.33 \mathrm{~dB}$ to coupling losses due to mode mismatch at the fiber-waveguide interface, estimated from a 1-D overlap integral between a

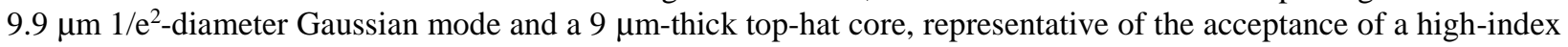
contrast, highly multimode waveguide. Assuming the additional insertion loss is entirely due to propagation loss, this places an upper limit on this value of $0.72 \mathrm{~dB}$ for the $9 \mathrm{~mm}$ guide or $0.80 \mathrm{~dB} / \mathrm{cm}$. To measure the gain of the system, the seed laser was switched into pulsed mode and the collimated waveguide output was reflected off a diffraction grating and focused onto an InGaAs photodiode. The amplitude of the signal was measured at different pump powers from 0 to $450 \mathrm{~mW}$ and is presented in Fig. 2. This procedure was performed for two seed wavelengths, $1533 \mathrm{~nm}$ and $1572 \mathrm{~nm} .1533 \mathrm{~nm}$ is where Er:YGG exhibits its peak emission cross-section but also suffers significant absorption resulting in pronounced three-level behavior and requiring a $47 \%$ upper-state population to overcome reabsorption and reach transparency (in the absence of other losses), this corresponds to 0 internal gain. For $1572 \mathrm{~nm}$, a $\mathrm{CO}_{2}$ absorption line, the significantly reduced thermal population of the lower laser level results in the requirement of only a $29 \%$ upper-state population to overcome reabsorption. Internal gain is achieved for both of these wavelengths, with $0.84 \mathrm{~dB} / \mathrm{cm}$ at $1533 \mathrm{~nm}$ (slightly exceeding the calculated upper limit on the propagation loss) and $0.44 \mathrm{~dB} / \mathrm{cm}$ internal gain at $1572 \mathrm{~nm}$. Due to the similar emission cross-sections at $1572 \mathrm{~nm}$ and $1651 \mathrm{~nm}$ for this material, this $0.44 \mathrm{~dB} / \mathrm{cm}$ is representative of the expected gains achievable at $1651 \mathrm{~nm}$ for similar pump and seed conditions.
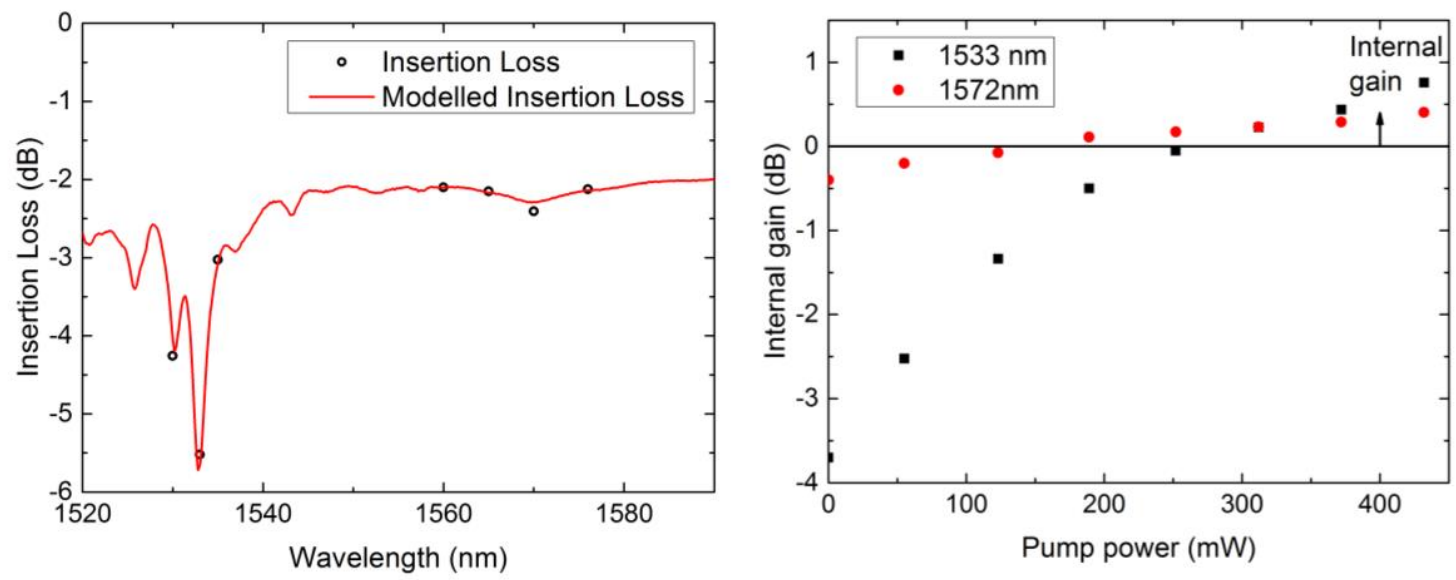

Fig. 2. (Left) Spectrally dependent insertion loss measurements and modelled insertion loss. (Right) Internal gain for the waveguide.

\section{Conclusions}

We have fabricated what we believe to be the first waveguide from the garnet Er:YGG. Initial characterization of the material shows spectroscopy well suited to LIDAR applications for the sensing of both methane and $\mathrm{CO}_{2}$. Internal gain has been realized in this system on the $1572 \mathrm{~nm} \mathrm{CO}$ LIDAR line and a similar amount of internal gain is expected at $1651 \mathrm{~nm}$. Further optimization of the growth conditions is expected to reduce propagation losses and further optimization of dopant concentration may lead to a significant increase in the achievable gain per unit length.

\section{References}

[1] T. Sakimura, Y. Watanabe, T. Ando, S. Kameyama, K. Asaka, H. Tanaka, T. Yanagisawa, Y. Hirano, H. Inokuchi. “3.2 mJ, 1.5 $\mu$ m laser power amplifier using an Er, Yb:glass planar waveguide for a coherent Doppler LIDAR,” Proc 17th Coherent Laser Radar Conference, Barcelona, Spain, (2013).

[2] J. A. Grant-Jacob, S. J. Beecher, T. L. Parsonage, P. Hua, J. I. Mackenzie, D. P. Shepherd, and R. W. Eason, "An 11.5 W Yb:YAG planar waveguide laser fabricated via pulsed laser deposition," Opt. Mater. Express 6, 91-96 (2016)

[3] H. Stange, K. Petermann, G. Huber, and E. W. Duczynski, "Continuous wave 1.6 um laser action in Er doped garnets at room temperature," Appl. Phys. B 49, 269-273 (1989).

[4] M. Kh. Ashurov, Yu. K. Voronko, V. V. Osiko, A. A. Sobol, and M. I. Timoshbchkin, "Spectroscopic study of stoichiometry deviation in crystals with garnet structure," Phys. Stat. Sol. 42, 101 (1977).

[5] T. L. Parsonage, S. J. Beecher, A. Choudhary, J. A. Grant-Jacob, P. Hua, J. I. Mackenzie, D. P. Shepherd, and R. W. Eason, "Pulsed laser deposited diode-pumped 7.4 W Yb: $\mathrm{Lu}_{2} \mathrm{O}_{3}$ planar waveguide laser," Opt. Express 23, 31691-31697 (2015). 